\title{
NONCOMMUTATIVE SYMMETRIC FUNCTIONS ASSOCIATED WITH A CODE, LAZARD ELIMINATION, AND WITT VECTORS
}

\author{
Jean-Gabriel Luque and Jean-Yves Thibon
}

IGM, Laboratoire d'informatique

77454 Marne-la-Vallée Cedex 2, France.

\{luque,jyt\}@univ-mlv.fr

July 11, 2006

\begin{abstract}
The construction of the universal ring of Witt vectors is related to Lazard's factorizations of free monoids by means of a noncommutative analogue. This is done by associating to a code a specialization of noncommutative symmetric functions.
\end{abstract}

Keywords: Witt vectors, codes, symmetric functions, factorizations of free monoids

\section{Introduction}

Among Ernst Witt's many contributions to mathematics, one finds two apparently unrelated ideas, both published in 1937 in two consecutive issues of Crelle's journal. The first one, the ring of Witt vectors [19] (a generalisation of $p$-adic numbers), solves a problem in commutative algebra, whilst the second one, the introduction of the free Lie algebra [20], definitely pertains to noncommutative mathematics. 
The aim of this note is to point out a close connection between both constructions, through the notion of noncommutative symmetric functions. What comes out is that the natural noncommutative analogues of the symmetric functions classically associated to the construction of universal Witt vectors can be related to another classical topic in the combinatorics of free Lie algebras: Lazard's factorizations of free monoids. This relation manifests itselfs when the elementary noncommutative symmetric functions are specialized by means of a code.

Our notations will be those of [11] and [7]. If $S$ is a set of words, we denote by $\underline{S}=\sum_{w \in S} w$ its characteristic series.

\section{Noncommutative Witt symmetric functions}

Let us first recall the construction of the universal ring $W(R)$ of Witt vectors associated to a commutative ring $R$. This is not Witt's original construction, but a simpler one that he found much later, and that he communicated to S. Lang, who published it as a series of exercises in his famous textbook of Algebra [9].

The ring $W(R)$ can be characterized by the following properties [4] (see also [8, 司):

(W1) As a set, $W(R)=\left\{\mathbf{a}=\left(a_{n}\right)_{n \geq 1}, a_{n} \in R\right\}$, and for any ring homomorphism $f: R \rightarrow R^{\prime}$, the map $W(f): \mathbf{a} \mapsto\left(f\left(a_{n}\right)\right)_{n \geq 1}$ is a ring homomorphism. $R$.

(W2) The maps $w_{n}: \mathbf{a} \mapsto \sum_{d \mid n} d a_{d}^{n / d}$ are ring homomorphisms $W(R) \rightarrow$

Operations on Witt vectors are better understood in terms of symmetric functions. Let $X=\left\{x_{n}, n \geq 1\right\}$ be an infinite set of commuting indeterminates (called here an alphabet), and following [14], define symmetric functions $q_{n}(X)$ by

$$
\prod_{n \geq 1} \frac{1}{1-t^{n} q_{n}(X)}=\sigma_{t}(X):=\sum_{n \geq 1} t^{n} h_{n}(X)
$$

where the complete homogeneous functions $h_{n}(X)$ are defined by $\sigma_{t}(X)=$ $\prod_{n \geq 1}\left(1-t x_{n}\right)^{-1}\left(c f\right.$. 11]). The $q_{n}$ 's are connected to the power-sums $p_{n}(X)=$ 
$\sum_{i} x_{i}^{n}$ by

$$
p_{n}=\sum_{d \mid n} d q_{d}^{n / d}
$$

and condition (W2) can be regarded as expressing the familiar properties of power sums

$$
\begin{gathered}
p_{n}(X+Y)=p_{n}(X)+p_{n}(Y) \\
p_{n}(X Y)=p_{n}(X) p_{n}(Y)
\end{gathered}
$$

where we use the $\lambda$-ring notation (an alphabet is identified with the formal sum of its elements). The transformation (11) is also used in [2, 12].

Reutenauer 14 studied the symmetric functions $q_{n}(X)$ and made the conjecture that for $n \geq 2,\left(-q_{n}\right)$ is Schur positive. This conjecture was proved in [17], in a generalised noncommutative form. Denoting as in [7] the noncommutative complete homogeneous symmetric functions of an alphabet $A$ by $S_{n}(A)$, one introduces noncommutative Witt symmetric functions $Q_{n}(A)$ by the identity

$$
\prod_{n \geq 1}^{\overrightarrow{1}} \frac{1}{1-t^{n} Q_{n}(A)}=\sigma_{t}(A):=\sum_{n \geq 1} t^{n} S_{n}(A)
$$

Then, it is proved in [17 that for $n \geq 2,\left(-Q_{n}\right)$ is a positive, multiplicity free, sum of noncommutative ribbon Schur functions $R_{I}$.

\section{Noncommutative symmetric functions as- sociated to a code}

Let $A$ be an alphabet and $C \subset A^{+}$a code, i.e. a minimal generating set of a free submonoid $C^{*}$ in $A^{*}$. Suppose we have a decomposition of $C$ as a disjoint union

$$
C=\coprod_{n \geq 1} C_{n}
$$

of possibly empty subsets $C_{n}$. In what follows, we shall in general assume that $C_{n}=C \cap A^{n}$, but this restriction in not necessary. In any case, we will consider that the elements of $C_{n}$ have degree $n$.

We will denote by $l(w)$ the length of a word $w$. Recall that, as a graded algebra, Sym $=\mathbb{Q}\left\langle\Lambda_{1}, \Lambda_{2}, \ldots\right\rangle$ where $\Lambda_{n}$ has degree $n$. We can define a 
specialization $\operatorname{Sym}[C]$ of the algebra of noncommutative symmetric functions by setting

$$
\Lambda_{n}[C]=(-1)^{n-1} \underline{C}_{n} .
$$

With this choice of signs, the complete symmetric functions are then given by

$$
S_{n}[C]=\sum_{w \in\left(C^{*}\right)_{n}} w
$$

the sum of all elements of degree $n$ in $C^{*}$. We call $C$-Witt symmetric function the value $Q_{i}[C]$ of $Q_{i}$ under this specialization. Let us give the first $Q_{i}[C]$ for three examples

Example 1 The Fibonacci prefix code $C=\{b, a b\}$.

$$
\begin{aligned}
& Q_{1}[C]=b \\
& Q_{2}[C]=a b \\
& Q_{3}[C]=a b^{2} \\
& Q_{4}[C]=a b^{3} \\
& Q_{5}[C]=a b^{2} a b+a b^{4} \\
& Q_{6}[C]=a b^{3} a b+a b^{5} \\
& Q_{7}[C]=a b^{6}+a b^{2}(a b)^{2}+a b^{4} a b+a b^{3} a b^{2}
\end{aligned}
$$

Example 2 The infinite prefix code $C=b a^{*}$.

$$
\begin{aligned}
& Q_{1}[C]=b \\
& Q_{2}[C]=b a \\
& Q_{3}[C]=b a^{2}+b a b \\
& Q_{4}[C]=b a b^{2}+b a^{3}+b a^{2} b \\
& Q_{5}[C]=b a b^{2} a+b a b^{3}+b a^{2} b^{2}+b a^{2} b^{2}+b a^{4}+b a^{2} b a \\
& Q_{6}[C]=b a^{4} b+b a^{3} b^{2}+b a^{2} b^{2} a+b a b^{3} a+b a b^{4}+b a^{3} b a+b a^{5}+b a^{2} b^{3}
\end{aligned}
$$

Example 3 The Dyck code $D$ (for the Dyck language $\underline{D^{*}}=1+a \underline{D^{*}} b \underline{D^{*}}$.)

$$
\begin{aligned}
& Q_{1}[D]=Q_{3}[D]=Q_{5}[D]=Q_{7}[D]=0 \\
& Q_{2}[D]=a b \\
& Q_{4}[D]=a a b b \\
& Q_{6}[D]=a a a b b b+a a b a b b+a a b b a b \\
& Q_{8}[D]=a a a a b b b b+a a a b a b b b+a a b a a b b b+a a a b b b a b+a a a b b a b b \\
& +a a b b a b a b+a a b a b a b b+a a b a b b a b
\end{aligned}
$$


On these examples, we remark that each $Q_{i}$ is multiplicity free and is the characteristic series of a code. In the following section, we prove that it is always the case and give a characterization of these codes in terms of Lazard's factorizations.

\section{Witt symmetric functions and factorization}

\subsection{Lazard elimination process}

We recall that a factorization of a monoid $\mathbb{M}$ is an ordered family of monoids $\mathbb{F}=\left(\mathbb{M}_{i}\right)_{i \in I}$ such that each element $m \in \mathbb{M}$ admits an unique decomposition

$$
m=m_{i_{1}} \cdots m_{i_{k}}
$$

where $i_{1}>\cdots>i_{k}$ and $m_{i_{1}} \in \mathbb{M}_{i_{1}}, \ldots, m_{i_{k}} \in \mathbb{M}_{i_{k}}$. In the case of free monoids $\mathbb{M}=A^{*}$, this property can be stated in terms of generating series

$$
\sum_{w \in A^{*}} w=\prod_{i} \sum_{w \in \mathbb{M}_{i}} w
$$

A submonoid $\mathbb{M}^{\prime} \subset \mathbb{M}$ can be characterized by its generating set $\mathbb{M}^{\prime} \backslash \mathbb{M}^{\prime 2}$. In the sequel, a factorization will be denoted by the sequence of the generating sets of its components, and for a factorization $\mathbb{F}=\left(C_{i}\right)_{i \in I}$ of $A^{*}$ into free submonoids, (8) reads

$$
\frac{1}{1-\underline{A}}=\prod_{i}^{\leftarrow} \frac{1}{1-\underline{C}_{i}} .
$$

Factorisations of free monoids have been extensively studied and the reader can refer to [3, 18] for a survey. A simple but relevant example is a Lazard bisection: considering a subalphabet $B \subset A$, one has

$$
A^{*}=B^{*}\left((A \backslash B) B^{*}\right)^{*} .
$$

The pair $\left(B,(A \backslash B) B^{*}\right)$ is a factorization of $A^{*}(c f$. [18, 3], and 15] for applicationa to free Lie algebras). Now, from (10) we can obtain a trisection (i.e. a factorization in three submonoids) by iterating the process on the left or the right factor, and so on. Factorisations which can be generated applying only Lazard bisections on the right factors will be called Lazard right compositions. This is a simple particular case of a locally finite right regular 
factorization [18. Let $A$ be an alphabet (possibly infinite) and $\omega: A \rightarrow \mathbb{N} \backslash 0$ a weight function. This function can be extended uniquely as a morphism $\omega: A^{*} \rightarrow(\mathbb{N},+)$. We can associate a factorization $\mathbb{F}(A, \omega)$ to this weight as follows. Let $\left(Z_{i}\right)_{i \geq 1}$ and $\left(C_{i}\right)_{i \geq 1}$ be the sequences of codes defined by the recurrence relations

1. $Z_{1}=A$

2. for each integer $i>0, C_{i}=Z_{i} \cap\left\{w \in A^{*} \mid \omega(w)=i\right\}$.

3. for each integer $i>0, Z_{i+1}=\left(Z_{i} \backslash C_{i}\right) C_{i}^{*}$

The sequence $\mathbb{F}(A, \omega)=\left(C_{i}\right)_{i \in I}$ obtained by omitting the empty elements in $\left(C_{i}\right)_{i \geq 1}$ is a Lazard right composition. Since each code $C \in A^{*}$ admits the length of words as weight function, we define the right length factorization $\mathbb{F}\left(C^{*}\right)$ of $C^{*}$ by

$$
\mathbb{F}\left(C^{*}\right)=\mathbb{F}(C, l) .
$$

We can remark that a code is homogeneous if and only if $\mathbb{F}\left(C^{*}\right)=(C)$.

\subsection{Computation of the $C$-Witt symmetric functions}

The equality between formal series (3) can be rewritten as

$$
\frac{1}{1-\underline{C}}=\frac{1}{1-Q_{1}} \frac{1}{1-Q_{2}} \frac{1}{1-Q_{3}} \cdots
$$

and in this section, we prove that this factorization of series is the weight right factorization of $C^{*}$.

In a more general setting, T. Scharf and one of the authors [17, gave a recursive algorithm for computing the $Q_{n}^{\prime} s$. Recall that the algebra $\mathbf{S y m}$ of noncommutative symmetric functions is the free associative algebra $\mathbb{Q}\left\langle S_{1}, S_{2}, \cdots\right\rangle$ generated by an infinite sequence of non commuting variables $S_{n}$, graded by the weight function $w\left(S_{n}\right)=n$. If $I=\left(i_{1}, \cdots, i_{n}\right)$, one defines

$$
\tilde{S}^{I}=(-1)^{n} S_{i_{1}} \cdots S_{i_{n}}
$$

The $Q_{i}$ can be computed following the rules given in [17]:

1. $F_{1}=-\sum_{i} \tilde{S}_{i}$,

2. $F_{n+1}=F_{n}+Q_{n}\left(1-F_{n}\right)$, 
3. $Q_{n}$ is the term of weight $n$ in $F_{n}$ multiplied by -1 .

Setting $Z_{n}=1-\left(1-F_{n}\right)^{-1}$, we obtain

$$
F_{n+1}=1-\left(1-Z_{n+1}\right)^{-1}
$$

and

$$
\begin{aligned}
F_{n}+Q_{n}\left(1-F_{n}\right) & =1-\left(1-Z_{n}\right)^{-1}+Q_{n}\left(1-Z_{n}\right)^{-1} \\
& =1-\left(1-Q_{n}\right)\left(1-Z_{n}\right)^{-1}
\end{aligned}
$$

This implies

$$
\begin{aligned}
Z_{n+1} & =1-\left(1-Z_{n}\right)\left(1-Q_{n}\right)^{-1} \\
& =\left(Z_{n}-Q_{n}\right)\left(1-Q_{n}\right)^{-1}
\end{aligned}
$$

Following [17], each $Q_{i}$ is multiplicity free on the $\tilde{S}_{I}$.

Let $Z_{i}[C]$ and $F_{i}[C]$ the values of $Z_{i}$ and $F_{i}$ under the specialization $S_{n}=S_{n}[C]$. The definition of noncommutative complete functions gives

$$
\sigma_{1}[C]=\sum S_{n}[C]=\frac{1}{1-\underline{C}}
$$

Hence, one has

$$
\begin{array}{r}
Z_{1}[C]=1-\left(\sigma_{1}[C]\right)^{-1}=\underline{C}, \\
Q_{1}[C]=S_{1}[C]=\underline{C_{1}}, \text { and } \\
Z_{1}[C]-Q_{1}[C]=\underline{C}-\underline{C_{1}} .
\end{array}
$$

It follows that $Z_{1}[C]$ and $Q_{1}[C]$ are the characteristic series of the codes $Z_{1}=C$ and $Q_{1}=C_{1}$. By induction, for each $n>0$ the series $Z_{n+1}[C]$ and $Q_{n+1}[C]$ are the characteristic series of $Z_{n+1}=\left(Z_{n} \backslash Q_{n}\right) Q_{n}^{*}$ and $Q_{n}^{*}=$ $Z_{n} \cap A^{\leq n}$. Hence, the following statement holds.

Proposition 1 Let $C$ be a code. Each $C$-Witt symmetric function $Q_{i}[C]$ is the characteristic series of a code, and the sequence obtained by deleting the empty set from $\left(Q_{1}, Q_{2}, \ldots, Q_{n}, \ldots\right)$ is the right length factorization of $C^{*}$.

Example 4 The sequence $\left(a, Q_{1}\left[b a^{*}\right], Q_{2}\left[b a^{*}\right], \ldots\right)$ is a factorization of $A^{*}$. The same method is applicable to compute homogeneous factorizations of 
non-homogeneous alphabets. For example, considering the alphabet $A=$ $\mathbb{N} \backslash 0$ with the weight $\omega=i d$, one finds

$$
\begin{aligned}
& Q_{1}[A]=1 \\
& Q_{2}[A]=2 \\
& Q_{3}[A]=21+3 \\
& Q_{4}[A]=211+31+4 \\
& Q_{5}[A]=2111+212+311+32+41+5 \\
& Q_{6}[A]=21111+51+2112+6+3111+312+42+411
\end{aligned}
$$

It is easy to see that this can be obtained from example 2 by the morphism $b a^{n} \rightarrow n+1$.

One has the following decomposition

Corollary 2 Let $w \in C^{*}$, then either $w \in C$ either there exist $w_{1}, w_{2} \in C^{*}$ such that $w=w_{1} w_{2}$, with $\omega\left(w_{2}\right)<\omega\left(w_{1}\right)<\omega(w)$. Furthermore, if $w_{1} \notin C$ then $w_{1}=w_{1}^{\prime} w_{1}^{\prime \prime}$ with $w_{1}^{\prime}, w_{1}^{\prime \prime} \in C^{*}$ and $\omega\left(w_{1}^{\prime \prime}\right) \leq \omega\left(w_{2}\right)$

This follows from the standard bracketing process of regular factorizations, which is described in [18].

\subsection{Noncommutative elementary symmetric functions and Lazard elimination}

The link between Lazard elimination and noncommutative Witt symmetric functions can be better understood in terms of elementary symmetric functions. The generating function of noncommutative elementary symmetric function is

$$
\lambda_{t}=\sum_{k \geq 0} \Lambda_{k} t^{k}
$$

These are related to noncommutative complete functions by

$$
\sigma_{t}=\frac{1}{\lambda_{-t}}=\frac{1}{1-\Lambda_{1} t+\Lambda_{2} t^{2}-\cdots+(-1)^{n} \Lambda_{n} t^{n}+\cdots} .
$$

If we set $\tilde{\Lambda}_{n}=(-1)^{n+1} \Lambda_{n}$, the series $\sigma_{1}$ can be considered as the characteristic series of the free monoid $\Lambda^{*}=\left\{\tilde{\Lambda}_{1}, \tilde{\Lambda}_{2}, \ldots, \tilde{\Lambda}_{n} \ldots\right\}^{*}$. We endow this monoid with the weight function defined by $\omega\left(\tilde{\Lambda}_{n}\right)=n$. Then, 
Theorem 3 One has

$$
\mathbb{F}(\boldsymbol{\Lambda}, \omega)=\left(Q_{1}[\boldsymbol{\Lambda}], Q_{2}[\boldsymbol{\Lambda}], \ldots\right)=\left(Q_{1}, Q_{2}, \ldots\right)
$$

This provides a simple algorithm for computing the decomposition of the noncommutative symmetric Witt functions on the basis of elementary symmetric functions. Let us gives the computation of the first $Q_{i}$ 's,

$$
\begin{aligned}
Q_{1}= & \Lambda_{1} \\
Q_{2}= & -\Lambda_{2} \\
Q_{3}= & -\Lambda_{2} \Lambda_{1}+\Lambda_{3} \\
Q_{4}= & -\Lambda_{2} \Lambda_{1} \Lambda_{1}+\Lambda_{3} \Lambda_{1}-\Lambda_{4} \\
Q_{5}= & -\Lambda_{2} \Lambda_{1}^{3}+\Lambda_{2} \Lambda_{1} \Lambda_{2}+\Lambda_{3} \Lambda_{1} \Lambda_{1}-\Lambda_{3} \Lambda_{2}-\Lambda_{4} \Lambda_{1}+\Lambda_{5} \\
Q_{6}= & -\Lambda_{2} \Lambda_{1}^{4}+\Lambda_{5} \Lambda_{1}+\Lambda_{2} \Lambda_{1} \Lambda_{1} \Lambda_{2}-\Lambda_{6}+\Lambda_{3} \Lambda_{1}^{3}-\Lambda_{3} \Lambda_{1} \Lambda_{2}+\Lambda_{4} \Lambda_{2} \\
& -\Lambda_{4} \Lambda_{1} \Lambda_{1} \\
Q_{7}= & -\Lambda_{3} \Lambda_{1}^{2} \Lambda_{2}+\Lambda_{3} \Lambda_{1}^{4}-\Lambda_{3} \Lambda_{1} \Lambda_{2} \Lambda_{1}+\Lambda_{3} \Lambda_{1} \Lambda_{3}+\Lambda_{3} \Lambda_{2}^{2}-\Lambda_{4} \Lambda_{1}^{3} \\
& +\Lambda_{4} \Lambda_{1} \Lambda_{2}-\Lambda_{4} \Lambda_{3}+\Lambda_{4} \Lambda_{2} \Lambda_{1}+\Lambda_{5} \Lambda_{1}^{2}-\Lambda_{5} \Lambda_{2}-\Lambda_{6} \Lambda_{1}+\Lambda_{7} \\
& -\Lambda_{2} \Lambda_{1}^{5}+\Lambda_{2} \Lambda_{1}^{3} \Lambda_{2}+\Lambda_{2} \Lambda_{1}^{2} \Lambda_{2} \Lambda_{1}-\Lambda_{2} \Lambda_{1}^{2} \Lambda_{3}-\Lambda_{2} \Lambda_{1} \Lambda_{2}^{2} \\
&
\end{aligned}
$$

(compare Examples 2 and 4 ).

The decomposition of the elementary functions on the basis $Q_{I}=Q_{i_{1}} \cdots Q_{i_{n}}$ is obtained by inspection of the series

$$
\frac{1}{\sigma_{t}}=\lambda_{-t}=1-\sum_{n} \tilde{\Lambda}_{n} t^{n}=\prod_{i}^{\leftarrow}\left(1-Q_{i} t^{i}\right) .
$$

One finds

$$
\tilde{\Lambda}_{n}=\sum_{k}(-1)^{k} \sum_{\substack{i_{1}>\cdots>i_{k} \\ i_{1}+\cdots+i_{k}=n}} Q_{i_{1}} \cdots Q_{i_{k}}
$$

\section{$5 \quad$ Examples involving Gaudier's *-multinomials}

\subsection{Gaudier's * multinomials}

Classical (commutative) Witt vectors give rise to examples involving interesting integer sequences. Let us recall for example the construction of $*$ multinomial coefficients and $*$-factorials given by Gaudier in 6 . If $R$ is a 
$\mathbb{Q}$-algebra, there is a commutative diagram

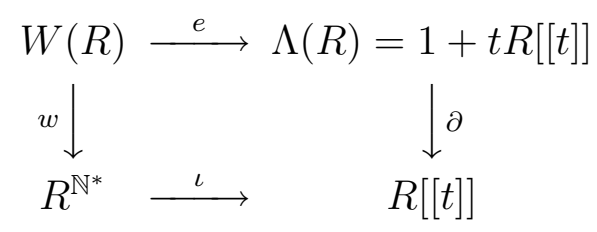

where

$$
\begin{gathered}
\partial=\frac{d}{d t} \ln \\
l\left(c_{1}, \cdots, c_{n}, \cdots\right)=\sum_{n \geq 1} c_{n} t^{n-1}, \\
w=\left(w_{1}, \cdots, w_{n}, \cdots\right), \\
e\left(a_{1}, \ldots, a_{n}, \ldots\right)=\prod_{\prod} \frac{1}{1-a_{n} t^{n}} .
\end{gathered}
$$

These maps are all isomorphisms. Let $i_{1}, i_{2}, \ldots, i_{k}$ be $k$ positive integers and $n=i_{1}+\cdots+i_{k}$. In [6] Gaudier has defined the $*$-multinomial coefficient $*\left(\begin{array}{c}n \\ i_{1}, \ldots, i_{k}\end{array}\right) \in W(\mathbb{Q})$ as the Witt vector such that $w_{p}=\left(\begin{array}{c}n p \\ p i_{1}, \ldots, p i_{k}\end{array}\right)$. In the same paper, he has defined the $*$-factorial $* n ! / n !$ by $w_{p}(* n ! / n !)=\frac{1}{n !} \frac{(p n) !}{p !^{n}}$. In particular, he has computed $e(* 2 ! / 2 !)=e\left(\frac{1}{2} *\left(\begin{array}{l}2 \\ 1\end{array}\right)\right)$ in closed form and related it to the Catalan numbers

$$
e(* 2 ! / 2 !)=\left(\frac{1+\sqrt{1-4 t}}{2}\right)^{-1}
$$

This raised the question whether it was possible to find similar expressions for other $*$-factorials and $*$-multinomials, and to give combinatorial interpretations.

\subsection{Non-commutative analogues of $\frac{1}{n} *\left(\begin{array}{l}n \\ 1\end{array}\right)$}

Consider the Dyck code of example 3. The free monoid $D^{*}$ is a submonoid of $\{a a, a b, b b\}^{*}$, graded by $\rho(a a)=\rho(b b)=\rho(a b)=1$. Under the $D$ specialization,

$$
\Lambda_{n}=(-1)^{n+1} \sum_{\substack{w \in D \\ \rho(w)=n}} w .
$$


Recall that the noncommutative power sums of the second kind $\Phi_{n}$ are defined by

$$
\sum_{n \geq 1} \Phi_{n} \frac{t^{n}}{n}=\log \sigma_{t}
$$

If one interprets Dyck words as binary trees, the $\Phi_{n}$ can be regarded as sums over forests

$$
\Phi_{n}=n \sum_{k} \frac{1}{k} \sum_{\substack{w_{1}, \ldots, w_{k} \in D \\ \rho\left(w_{1}\right)+\cdots+\rho\left(w_{k}\right)=n}} w_{1} \cdots w_{k} .
$$

Example 5.1 The first values of $\Phi_{n}$ are

$$
\begin{aligned}
& \Phi_{1}=a b \\
& \Phi_{2}=2 a a b b+a b \cdot a b \\
& \Phi_{3}=3 a a a b b b+3 a a b a b b+\frac{3}{2} a a b b \cdot a b+\frac{3}{2} a b \cdot a a b b+a b \cdot a b \cdot a b \\
& \Phi_{4}=4 a a a a b b b b+4 a a a b b a b b+4 a a b a a b b b+4 a a b a b a b b+4 a a a b a b b b \\
& \quad+2 a a a b b b \cdot a b+2 a a b a b b \cdot a b+2 a b \cdot a a a b b b+2 a b \cdot a a b a b b+\frac{4}{3} a a b b \cdot a b \cdot a b \\
& \quad+\frac{4}{3} a b \cdot a a b b \cdot a b+\frac{4}{3} a b \cdot a b \cdot a a b b+a b \cdot a b \cdot a b \cdot a b
\end{aligned}
$$

See example 3 for the first values of $Q_{n}$.

Setting $\pi(w)=1$ for all words $w$, one has

$$
\pi\left(\Phi_{n}\right)=w_{n}\left(\frac{1}{2 !} * 2 !\right)=w_{n}\left(\frac{1}{2} *\left(\begin{array}{l}
2 \\
1
\end{array}\right)\right)
$$

and one recovers (26).

More generally, for $* \frac{1}{n}\left(\begin{array}{c}n \\ 1\end{array}\right)$, an easy application of the Lagrange inversion formula gives that

$$
e\left(\frac{1}{n} *\left(\begin{array}{c}
n \\
1
\end{array}\right)\right)=\sum_{k \geq 0} \frac{\left(\begin{array}{c}
n k \\
k
\end{array}\right)}{(n-1) k+1} t^{k}
$$

is also the generating series of $n$-ary trees. Consider the free monoid $F_{n}=T_{n}^{*}$ over the alphabet $T_{n}=\left\{a_{\mathbf{t}}\right\}$ whose letters are labelled by $n$-ary trees and weighted by $\rho\left(a_{\mathbf{t}}\right)=E(\mathbf{t}) / n$, where $E(\mathbf{t})$ is the number of edges of $\mathbf{t}$. Instead of specializing $\Lambda_{k}$ as in the case $n=2$, we set $S_{k}=\sum_{\rho(\mathbf{t})=k} a_{\mathbf{t}}$. Then, we obtain

$$
\Phi_{k}=k \sum_{p} \frac{(-1)^{p+1}}{p} \sum_{\substack{\mathbf{t}_{1}, \ldots, \mathbf{t}_{p} \\ \rho\left(\mathbf{t}_{1}\right)+\cdots+\rho\left(\mathbf{t}_{p}\right)=k}} a_{\mathbf{t}_{1}} \cdots a_{\mathbf{t}_{p}} .
$$


Again, applying the morphism $\pi(w)=1$, we obtain

$$
\pi\left(\Phi_{k}\right)=w_{k}\left(\frac{1}{n} *\left(\begin{array}{c}
n \\
1
\end{array}\right)\right)
$$

that is, we recover (31). Remark that this implies the identity

$$
\sum_{p} \frac{(-1)^{p+1}}{p} \sum_{i_{1}+\cdots+i_{p}=k} \prod_{m} \frac{\left(\begin{array}{c}
n i_{m} \\
i_{m}
\end{array}\right)}{(n-1) i_{m}+1}=\frac{1}{n k}\left(\frac{n k}{k}\right) .
$$

\subsection{Combinatorial interpretations of some $*$-binomial coefficients}

Remark that, as in section 5.2, the specialization $S_{k}=\sum_{\rho(\mathbf{t})=k+1} a_{\mathbf{t}}$ gives a non-commutative analogue of

$$
e\left(*\left(\begin{array}{c}
n \\
1
\end{array}\right)\right)(z)=\sum_{k=1}^{\infty} \frac{\left(\begin{array}{c}
n k \\
k
\end{array}\right)}{(n-1) k+1} t^{k-1}=\frac{e\left(\frac{1}{n} *\left(\begin{array}{c}
n \\
1
\end{array}\right)\right)-1}{z} .
$$

This last equality can be generalized as follows.

Proposition 5.2 Let $p \geq 1$ and $\omega=e^{2 i \pi / p}$.

$$
\begin{aligned}
e\left(*\left(\begin{array}{c}
n p \\
p
\end{array}\right)\right) & =\frac{-1}{z} \prod_{k=0}^{p-1}\left(1-e\left(\frac{1}{n} *\left(\begin{array}{c}
n \\
1
\end{array}\right)\right)\left(\omega^{k} z^{\frac{1}{p}}\right)\right) \\
& =\prod_{k=0}^{p-1} e\left(*\left(\begin{array}{c}
n \\
1
\end{array}\right)\right)\left(\omega^{k} z^{\frac{1}{p}}\right)
\end{aligned}
$$

Proof We first prove (37), by computing

$$
\begin{aligned}
\ln \left(\prod_{k=0}^{p-1} e\left(*\left(\begin{array}{c}
n \\
1
\end{array}\right)\right)\left(\omega^{k} z^{\frac{1}{p}}\right)\right) & = \\
\sum_{k=0}^{p-1} \ln \left(e\left(*\left(\begin{array}{c}
n \\
1
\end{array}\right)\right)\left(\omega^{k} z^{\frac{1}{p}}\right)\right) & = \\
\sum_{k=0}^{p-1} \sum_{j \geq 1} \frac{\left(\begin{array}{c}
j n \\
j
\end{array}\right)}{j} \omega^{j k} z^{\frac{j}{p}} & =\sum_{k \geq 1} \frac{\left(\begin{array}{c}
k n p \\
k^{p}
\end{array}\right)}{k} z^{k} \\
& =\ln \left(e\left(*\left(\begin{array}{c}
n p \\
p
\end{array}\right)\right) .\right.
\end{aligned}
$$


Next (36) follows from the equality

$$
e\left(*\left(\begin{array}{c}
n p \\
p
\end{array}\right)\right)=\prod_{k=0}^{p-1} \frac{e\left(\frac{1}{n} *\left(\begin{array}{c}
n \\
1
\end{array}\right)\right)\left(\omega^{k} z^{\frac{1}{p}}\right)-1}{\omega^{k} z^{\frac{1}{p}}}
$$

¿From [13] (Theorem 1, p.7), the series $e\left(*\left(\begin{array}{c}n p \\ p\end{array}\right)\right)$ is the generating series of the number $q_{m}$ of lattice paths from $(0,0)$ to $((n-1) p m, p m)$ that never go above the path $\left(\uparrow^{(n-1) p} \rightarrow^{p}\right)^{m}$ (lattice paths are represented by words over the alphabet $F=\{\rightarrow, \uparrow\}$, where $\rightarrow$ means the elementary horizontal path $(1,0)$ and $\uparrow$ the elementary vertical path $(0,1))$.

Example 5.3 The series

$$
\begin{aligned}
e\left(*\left(\begin{array}{c}
4 \\
2
\end{array}\right)\right)(z) & =1+6 z+53 z^{2}+554 z^{3}+6362 z^{4}+\cdots \\
& =e\left(*\left(\begin{array}{l}
2 \\
1
\end{array}\right)\right)\left(z^{\frac{1}{2}}\right) e\left(*\left(\begin{array}{l}
2 \\
1
\end{array}\right)\right)\left(-z^{\frac{1}{2}}\right)
\end{aligned}
$$

is also the generating series of ordered trees on $2 n$ nodes with every subtree at the root having an even number of edges(see Sloane [16] ID number: A066357). See also [1] for another enumeration.

$$
\begin{aligned}
e\left(*\left(\begin{array}{c}
6 \\
2
\end{array}\right)\right)(z)= & 1+15 z+360 z^{2}+10463 z^{3}+\cdots \\
= & e\left(*\left(\begin{array}{c}
3 \\
1
\end{array}\right)\right)\left(z^{\frac{1}{2}}\right) e\left(*\left(\begin{array}{l}
3 \\
1
\end{array}\right)\right)\left(-z^{\frac{1}{2}}\right) \\
e\left(*\left(\begin{array}{c}
6 \\
3
\end{array}\right)\right)(z)= & 1+20 z+662 z^{2}+26780 z^{3}+\cdots \\
= & e\left(*\left(\begin{array}{c}
2 \\
1
\end{array}\right)\right)\left(z^{\frac{1}{3}}\right) e\left(*\left(\begin{array}{l}
2 \\
1
\end{array}\right)\right)\left(\exp \left\{\frac{2 i \pi}{3}\right\} z^{\frac{1}{3}}\right) \times \\
& \times e\left(*\left(\begin{array}{c}
2 \\
1
\end{array}\right)\right)\left(\exp \left\{\frac{4 i \pi}{3}\right\} z^{\frac{1}{3}}\right) \\
e\left(*\left(\begin{array}{c}
12 \\
3
\end{array}\right)\right)(z)== & 1+220 z+91498 z^{2}+47961320 z^{3}+\cdots \\
= & e\left(*\left(\begin{array}{c}
4 \\
1
\end{array}\right)\right)\left(z^{\frac{1}{3}}\right) e\left(*\left(\begin{array}{l}
4 \\
1
\end{array}\right)\right)\left(\exp \left\{\frac{2 i \pi}{3}\right\} z^{\frac{1}{3}}\right) \times \\
& \times e\left(*\left(\begin{array}{c}
4 \\
1
\end{array}\right)\right)\left(\exp \left\{\frac{4 i \pi}{3}\right\} z^{\frac{1}{3}}\right)
\end{aligned}
$$

One can construct noncommutative analogues of the $*\left(\begin{array}{c}n p \\ p\end{array}\right)$ by specializing $S_{m}$ to the sum of the words coding the lattice paths from $(0,0)$ to $((n-1) m p, m p)$ that never go above the path $\left(\uparrow^{(n-1) p} \rightarrow^{p}\right)^{m}$.

Example 5.4 Let us consider the non-commutative analogue of $*\left(\begin{array}{l}2 \\ 1\end{array}\right)$. Under this specialization, the first values of the $S_{n}$ are

$$
\begin{aligned}
& S_{1}=\uparrow \rightarrow+\rightarrow \uparrow \\
& S_{2}=\rightarrow^{2} \uparrow^{2}+(\rightarrow \uparrow)^{2}+\rightarrow^{2} \rightarrow+\uparrow \rightarrow^{2} \uparrow+(\uparrow \rightarrow)^{2}
\end{aligned}
$$




$$
\begin{aligned}
S_{3}= & \rightarrow^{3} \uparrow^{3}+\rightarrow^{2} \uparrow \rightarrow^{2}+\rightarrow^{2} \uparrow^{2} \rightarrow \uparrow+\rightarrow^{2} \uparrow^{3} \rightarrow \\
& +\rightarrow \uparrow \rightarrow^{2} \uparrow^{2}+(\rightarrow \uparrow)^{3}+(\rightarrow \uparrow)^{2} \uparrow \rightarrow+\uparrow^{2} \rightarrow^{2} \uparrow \\
& +\rightarrow \uparrow^{2} \rightarrow \uparrow \rightarrow+\uparrow \rightarrow^{3} \uparrow^{2}+\uparrow \rightarrow^{2} \uparrow \rightarrow \uparrow+\uparrow^{2} \uparrow^{2} \rightarrow+\uparrow \rightarrow \uparrow \rightarrow^{2} \uparrow+(\uparrow \rightarrow)^{3}
\end{aligned}
$$

and the first values of the $\Lambda_{n}$ are

$$
\begin{aligned}
& \Lambda_{1}=\uparrow \rightarrow+\rightarrow \uparrow \\
& \Lambda_{2}=-\rightarrow^{2} \uparrow^{2} \\
& \Lambda_{3}=\rightarrow^{2 \uparrow} \rightarrow^{2}+\rightarrow^{3} \uparrow^{3}
\end{aligned}
$$

More generally, from

$$
\Lambda_{m}=(-1)^{m+1} \sum_{k=1}^{m}(-1)^{k} \sum_{i_{1}+\cdots+i_{k}=m} S_{i_{1}} \cdots S_{i_{k}}
$$

one obtains

$$
\Lambda_{m}=(-1)^{m+1} \sum_{w} w
$$

where the sum is over all lattice paths from $(0,0)$ to $((n-1) m p, m p)$ that never go above $\left(\uparrow^{(n-1) p} \rightarrow^{p}\right)^{m}$ and which avoid the points $((n-1) k p, k p)$ for $0<k<m$. Hence,

$$
\Phi_{m}=m \sum_{w} \frac{w}{c(w)+1}
$$

where the sum is over the lattice paths from $(0,0)$ to $((n-1) m p, m p)$ below $\left(\uparrow^{(n-1) p} \rightarrow^{p}\right)^{m}$, and $c(w)$ is the number of points $((n-1) k p, k p)$ belonging to the path $w$, with $0<k<m$.

Example 5.5 Let us consider the noncommutative analogue of $*\left(\begin{array}{l}4 \\ 2\end{array}\right)$. The first values of the $\Lambda_{n}$ are

$$
\begin{aligned}
& \Lambda_{1}=\rightarrow^{2} \uparrow^{2}+(\rightarrow \uparrow)^{2}+\rightarrow^{2} \rightarrow+\uparrow \rightarrow^{2} \uparrow+\uparrow \rightarrow \uparrow \rightarrow+\uparrow^{2} \rightarrow^{2} \\
& \Lambda_{2}=-\left(\rightarrow^{4} \uparrow^{4}+\rightarrow^{3} \uparrow \rightarrow \uparrow^{3}+\rightarrow^{3} \uparrow^{2} \rightarrow \uparrow^{2}+\rightarrow^{3} \uparrow^{3} \rightarrow \uparrow+\rightarrow^{3} \uparrow^{4} \rightarrow\right. \\
& +\rightarrow^{2 \uparrow} \rightarrow^{2} \uparrow^{3}+\rightarrow^{2 \uparrow}(\rightarrow \uparrow)^{2} \uparrow+\rightarrow^{2} \uparrow \rightarrow^{2} \rightarrow \uparrow+\rightarrow^{2} \uparrow \rightarrow \uparrow^{3} \rightarrow \\
& +\rightarrow \uparrow \rightarrow^{3} \uparrow^{3}+\rightarrow \uparrow \rightarrow^{2} \uparrow \rightarrow \uparrow^{2}+\rightarrow \uparrow \rightarrow \uparrow^{2} \rightarrow \uparrow+\rightarrow \uparrow \rightarrow \uparrow^{3} \rightarrow \\
& \left.+\uparrow \rightarrow^{4} \uparrow^{3}+\uparrow \rightarrow^{3} \uparrow \rightarrow^{2}+\uparrow \rightarrow^{2} \uparrow^{2} \rightarrow \uparrow+\uparrow \rightarrow^{2} \uparrow^{3} \rightarrow\right)
\end{aligned}
$$

A natural question is whether it is possible to find similar interpretations for other $*$-binomials. 


\subsection{Shuffle analogues of $*$-multinomials in non commu- tative Witt vectors}

We shall now construct another noncommutative analogue of $*$-multinomials. Let $A$ be an alphabet and $w_{1}, \cdots, w_{k}$ be $k$ words of respective lengths $m_{1}, \ldots, m_{k}$. Let $w=w_{1} \cdot w_{2} \cdots w_{k}$ and $m=m_{1}+\cdots+m_{k}$. The shuffle $w_{1} \sqcup \cdots \sqcup w_{k}$ contains $\left(\begin{array}{c}m \\ m_{1}, \ldots, m_{k}\end{array}\right)$ terms, and we can denote it by $\left(\begin{array}{c}w \\ w_{1}, \ldots, w_{k}\end{array}\right)$ to emphasize this point. Then, we introduce the noncommutative Witt vector analogue

$$
*\left(\begin{array}{c}
w \\
w_{1}, \ldots, w_{k}
\end{array}\right)=\left(Q_{1}, Q_{2}, \cdots, Q_{n}, \cdots\right)
$$

given by the sequence of Witt symmetric functions under the specialization

$$
\Phi_{n}=w_{1}^{n} \sqcup w_{2}^{n} \sqcup \cdots \sqcup w_{k}^{n} .
$$

Example 5.6 If $w_{i}=a^{p_{i}}$, one has

$$
\begin{gathered}
*\left(\begin{array}{c}
w \\
w_{1}, \ldots, w_{k}
\end{array}\right)=\left(w _ { 1 } \left(*\left(\begin{array}{c}
p_{1}+\cdots+p_{k} \\
p_{1}, \ldots, p_{k}
\end{array}\right) a^{p_{1}+\cdots+p_{k}}\right.\right. \\
\cdots, w_{n}\left(*\left(\begin{array}{c}
\left(p_{1}+\cdots+p_{k}\right) \\
p_{1}, \ldots, p_{k}
\end{array}\right) a^{n\left(p_{1}+\cdots+p_{k}\right)}, \cdots\right) .
\end{gathered}
$$

Sending $a$ to 1 , one recovers $*$-multinomials.

\section{Acknowledgements}

The authors are grateful to Henri Gaudier for fruitful discussions and comments.

This project has been partially supported by EC's IHRP Programme, grant HPRN-CT-2001-00272, "Algebraic Combinatorics in Europe".

\section{References}

[1] C. Banderier, D. Merlini, Lattice paths with an infinite set of jumps, Proc. Formal Power Series and Algebraic Combinatorics 2002, The University of Melbourne, Australia. 
[2] F. Bassino, M.-P. BÉAl, D. Perrin, Length distributions and regular sequences, in Codes, Systems ans Graphical Models, IMA Volumes in Mathematics and its Applications, Springer-Verlag, p. 415-437, 2001.

[3] J. Berstel, D. Perrin, Theory of Codes, Academic Press, New-York, 1985 .

[4] P. CArtier, Groupes formels associés aux anneaux de Witt généralisés, C.R. Acad. Sci. Paris 265 (1967), 49-52.

[5] J. Dieudonné, Schur functions and group representations, Astérisque 87-88 (1983), 7-19.

[6] H. Gaudier, Relévement des coefficients binômiaux dans les vecteurs de Witt, Séminaire Lotharingien de Combinatoire 16 (1988), 93-108.

[7] I.M. Gelfand, D. Krob, A. Lascoux, B. Leclerc, V.S. Retakh, J.-Y. Thibon, Noncommutative symmetric functions, Adv. Math. 112 (1995), 218-348.

[8] M. Hazewinkel, Formal Groups and Applications, Academic Press, New York, 1978.

[9] S. Lang, Algebra. Addison-Wesley Publishing Co., Inc., Reading, Mass. 1965.

[10] M. LAzARD, Groupes, anneaux de Lie et problème de Burnside, Istituto Matematico dell'Università di Roma, 1960.

[11] I.G. Macdonald, Symmetric functions and Hall polynomials, Oxford Math. Monographs, Oxford, 1979; 2nd ed. 1995.

[12] N. Metropolis, G.-C. Rota, Witt vectors and the algebra of necklaces, Adv. Math. 50 (1983), 95-125.

[13] A. De Mier, M. Noy, A solution to the tennis ball problem, Proc. Formal Power Series and Algebraic Combinatoricsi 2004, University of British Columbia (Vancouver B.C., Canada).

[14] C. Reutenauer, On symmetric functions related to Witt vectors and the free Lie algebra, Adv. Math. 110 (1995), 234-246. 
[15] C. Reutenauer, Free Lie Algebras. Clarendon Press, Oxford, 1993.

[16] N. J. A. Sloane, The On-Line Encyclopedia of Integer Sequences, http://www.research.att.com/ njas/sequences/

[17] T. Scharf, J.-Y. Thibon, On Witt vectors and symmetric functions, Algebra Colloq. 3 (1996), 231-238.

[18] G. Viennot, Algèbres de Lie libres et monö̈des libres, Thèse de Doctorat d'Etat, Université Paris VII, 1974.

[19] E. WiTT, Zyklische Körper und Algebren der Charakteristik $p$ vom Grade $p^{m}$, J. reine angew. Math. 176 (1937), 126-140.

[20] E. WiTt, Treue Darstellung Liescher Ringe, J. reine angew. Math. 177 (1937), 152-160. 